\title{
Spin-Flip Transitions in Tunneling through a Quantum Dot Coupled to Ferromagnetic Electrodes
}

\author{
W. RUDZIŃSKI \\ Department of Physics, Adam Mickiewicz University \\ Umultowska 85, 61-614 Poznań, Poland
}

\begin{abstract}
Using nonequilibrium Green function formalism the spin-polarized electronic transport through a quantum dot coupled to ferromagnetic electrodes is studied. Arbitrary Coulomb correlations and spin-flip processes are considered in the dot, for both parallel and antiparallel magnetic configurations of the junction. It is found that spin-flip processes suppress the magnetoresistance between each pair of the threshold bias voltages, thus giving rise to sharp tunnel magnetoresistance maxima at the thresholds. The origin of additional steps in the electric current, splitting of the resonance peaks in the corresponding differential conductance as well as negative differential conductance is also discussed in the context of interplay between tunneling processes and the intradot spin-flip transitions.
\end{abstract}

PACS numbers: 72.25.Mk, 73.63.Kv, 73.23.Hk

\section{Introduction}

During the last decade, the prospective development of spintronic devices for the storage, transport and precessing of information has stimulated experimental research on spin manipulation and understanding of electron spin relaxation processes in semiconductor or metallic granular mesoscopic structures (see e.g. [1-3] and references therein). Theoretical investigations were addressed mostly to spin-flip scattering in a context of electric current tunneling through a single-level quantum dot (QD) coupled to ferromagnetic external electrodes. One of the most widely studied spin-dependent effects in the devices considered here is the tunnel magnetoresistance (TMR) defined quantitatively as $\mathrm{TMR}=\left(J_{\mathrm{P}}-J_{\mathrm{AP}}\right) / J_{\mathrm{AP}}$, with $J_{\mathrm{P}}$ and $J_{\mathrm{AP}}$ denoting electric current in the parallel $(\mathrm{P})$ and antiparallel (AP) magnetic configuration of the external electrodes, respectively. It is found that the presence of the spin-flip process in the dot suppresses TMR in the sequential tunneling regime and reduces the magnetically induced asymmetry in the current-voltage characteristics with respect to the bias reversal [4]. It is also shown that the intradot spin-flip transitions result in a splitting of the Kondo peak into three well defined peaks and that linear TMR may be inverted at the Kondo resonance [5]. Inversion of TMR has been also observed above the Kondo temperatures, in the cotunneling regime analyzed for fast intrinsic spin relaxation on the dot [6]. In the most recent calculation [7] it is reported that in the AP configuration spin-flip can result in a switch of the tunneling current polarization from the source to the drain lead and may lead to a significant suppression of the Fano factor.

The present paper extends previous studies reported in Refs. [4, 7] and here an attempt at investigating nonequilibrium features of TMR within nonequilibrium Green function approach is proposed. Hence, TMR phenomenon is studied in the context of the both sequential as well as higher order tunneling processes in a magnetic tunnel junction based on a single-level QD with assumed nonvanishing intradot Coulomb correlations and spin-flip transitions. Our discussion deals with the electronic transport above the Kondo regime.

\section{Model and method}

In the model Hamiltonian of the system, the left and right ferromagnetic electrodes are taken in the noninteracting quasiparticle limit. The term corresponding to the dot includes the single particle energy level $\varepsilon_{d}$, Coulomb correlations described by the Hubbard parameter $U$ as well as the part which accounts for spin-flip of the electron in the dot with the $R$ parameter denoting the spin-flip rate. Since in the presence of intradot spin relaxation processes electrons tunnel into a superposition of spin-up and spin-down states, then this coherence technically may be introduced by a spin rotation transformation in terms of which the dot Hamiltonian is reduced to two terms: the Hubbard one and the term including the single particle energy level $\varepsilon_{d} \pm R$ with the plus and minus sign for minority (down) and majority (up) spins, respectively. Spin asymmetry of the tunneling rates across the left and right barriers is described by the parameters $p_{1}$ and $p_{\mathrm{r}}$. To calculate the occupation numbers of the dot we used nonequilibrium Green-function method based on equation of motion in the Hartree-Fock approximation. Having found self-consistently the occupation numbers, one can calculate the tunneling current from the MeirWingreen formula [8]. 


\section{Numerical results}

Let us consider features of the electronic transport through a symmetrical tunnel junction, i.e. assume that the both barriers are identical and the dot is coupled to two identical ferromagnetic electrodes, $p_{\mathrm{l}}=p_{\mathrm{r}}$. The dot energy level is assumed to be empty in the equilibrium situation, $\varepsilon_{d}>0$ at $V=0$.

Bias dependence of electric current, differential conductance as well as the corresponding TMR for such a system in the absence of spin-flip processes on the dot, $R=0$, are shown in Fig. $1 \mathrm{a}-\mathrm{c}$. The current curves in Fig. 1a have the typical step-like profile with two steps appearing at threshold bias voltages at which the dot energy levels $\varepsilon_{d}$ and $\varepsilon_{d}+U$ cross the Fermi level of the source electrode. Thus, the corresponding differential conductance in Fig. 1b exhibits two resonance peaks. The difference in spin asymmetry in the tunneling rates across the left and right barriers in the antiparallel configuration gives rise to inequality in occupation numbers for up and down-spin electrons on the dot. No such effect occurs in the parallel configuration for which the spin asymmetry for both barriers is the same. Hence, as shown in Fig. 1a, differences between the sequential tunneling current in the $\mathrm{P}$ and AP configuration appear, which consequently give rise to the displayed in Fig. 1c enhancement of the TMR ratio between the two thresholds. In turn, the central flat TMR maximum in Fig. 1c appears at the bias voltage range, where the sequential tunneling current is exponentially suppressed and arises due to higher-order tunneling processes.

If we include spin-flip transitions, then the difference between up- and down-spin occupation numbers in the AP configuration is reduced and as clearly seen in Fig. 1d, the electric current $J_{\mathrm{AP}}$ calculated for the AP alignment of the lead magnetizations tends to overlap the electric current $J_{\mathrm{P}}$ in the $\mathrm{P}$ configuration. On the other hand, since spin relaxation processes lift the degeneracy of the dot discrete level, additional steps in the current are observed at the threshold bias voltages. In particular, when the dot is singly occupied in the $\mathrm{P}$ configuration, a small bump in the $J_{\mathrm{P}}$ characteristics appears above the first threshold bias voltage. The latter property may be explained as follows. When the spin channel $\varepsilon_{d}-R$ enters the tunneling window, then the majority electrons give rise to the current enhancement at $V>2\left(\varepsilon_{d}-R\right)$. This tendency holds until the channel $\varepsilon_{d}+R$ is not active in sequential tunneling. If the channel $\varepsilon_{d}+R$ enters the tunneling window, then also the minority electrons may be transmitted sequentially through the junction. Since in the $\mathrm{P}$ configuration the minority states in a drain electrode are filled up with a smaller probability than the majority ones, then an interplay between the spin-flip and tunneling rates leads to a suppression of the tunneling current at bias voltages larger than $2\left(\varepsilon_{d}+R\right)$. No such behavior occurs in the AP configuration, as in symmetric junctions the product of transmission rates across the left and right barrier is the same for the majority and minority electrons. Figure 1d shows also that due to

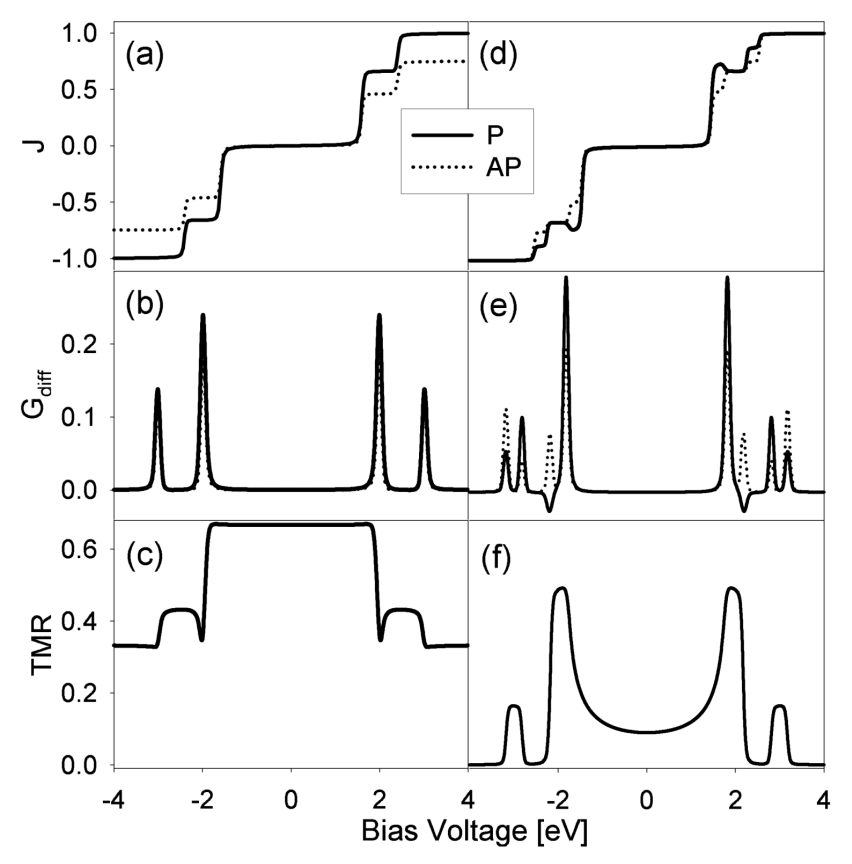

Fig. 1. Bias dependence of the electric current $J$, differential conductance $G_{\text {diff }}$ and TMR for spin-flip rates $R=0 \mathrm{eV}$ (a)-(c) and $R=0.09 \mathrm{eV}$ (d)-(f). The parameters are $p_{1}=p_{\mathrm{r}}=0.5, \varepsilon_{d}=1 \mathrm{eV}, U=0.5 \mathrm{eV}$ and $T=100 \mathrm{~K}$.

the intradot Coulomb repulsion the dot may be doubly occupied and thus electrons may tunnel through two additional spin channels $\varepsilon_{d}+U \pm R$. Consequently, a further enhancement of the quantity $J_{\mathrm{P}(\mathrm{AP})}$ around the second threshold bias voltage is observed, with current steps appearing at $V=2\left(\varepsilon_{d}+U-R\right)$ and $V=2\left(\varepsilon_{d}+U+R\right)$. The discussed above features of electric current give rise to splitting of the resonance peaks of the differential conductance (see Fig. 1e). Each pair of the split conductance peaks is separated at the distance depending on the strength of the spin-flip rate $R$. It is also seen that the mechanism responsible for occurrence of the bump in the current in the $\mathrm{P}$ configuration results in a negative differential conductance with a sharp minimum at $V=2\left(\varepsilon_{d}+R\right)$.

Finally, Fig. If shows that spin-flip transitions suppress the displayed in Fig. 1c maxima of the corresponding TMR. More specifically, when $R>0$, the TMR maxima between the two thresholds are entirely reduced, while at bias voltages $\varepsilon_{d}+R<\frac{V}{2}<\varepsilon_{d}-R$ and $\varepsilon_{d}+U+R<\frac{V}{2}<\varepsilon_{d}+U-R$ the TMR ratio is significantly enhanced. One may readily deduce from Fig. 1d and e that the width of these TMR peaks equals $4 R$. Besides, when $R>0$ the central plateau in TMR is reshaped into a dip with a non-zero minimum at $V=0$. The latter indicates that even in the presence of intradot spin-flip transitions higher order tunneling processes may contribute to electronic transport in the bias voltage regime for which sequential tunneling is exponentially suppressed. 


\section{Conclusions}

The present work reveals that intradot spin-flip transitions give rise to additional steps in electric current at transport voltage range around threshold bias voltages. Consequently, as a result of electron transitions across tunneling barriers into a superposition of spin-up and spin-down states on the dot, the resonance peaks in the corresponding differential conductance are split into two peaks. It is also found that due to interplay between the tunneling and spin-flip rates a negative differential conductance in the vicinity of the first threshold bias voltage may be observed. At bias voltages sufficiently far from the thresholds the spin relaxation processes reduce the difference between the majority and minority dot occupation numbers, and the AP electric current tends to overlap the current characteristics obtained for the $\mathrm{P}$ configuration. The discussed current properties result in significant suppression of the TMR ratio in the whole bias voltage range except for the resonance region at which TMR maxima appear.

\section{Acknowledgments}

This work was supported by funds of the Polish Ministry of Science and Higher Education as a research project in years 2006-2009.

\section{References}

[1] J.M. Elzerman, R. Hanson, L.H. Willems van Beveren, B. Witkamp, L.M.K. Vandersypen, L.P. Kouwenhoven, Nature 430, 431 (2004).

[2] L.Y. Zhang, C.Y. Wang, Y.G. Wei, X.Y. Liu, D. Davidović, Phys. Rev. B 72, 155445 (2005).

[3] T. Meunier, I.T. Vink, L.H. Willems van Beveren, K.-J. Tielrooij, R. Hanson, F.H.L. Koppens, H.P. Tranitz, W. Wegscheider, L.P. Kouwenhoven, L.M.K. Vandersypen, Phys. Rev. Lett. 98, 126601 (2007).

[4] W. Rudziński, J. Barnas, Phys. Rev. B 64, 085318 (2001).

[5] Ping Zhang, Qi-Kun Xue, Yupeng Wang, X.C. Xie, Phys. Rev. Lett. 89, 286803 (2002).

[6] I. Weymann, J. Barnaś, Phys. Rev. B 73, 205309 (2006).

[7] F.M. Souza, A.P. Jauho, J.C. Egues, cond-mat 0802.0982 (2008).

[8] A.-P. Jauho, N.S. Wingreen, Y. Meir, Phys. Rev. B 50, 5528 (1994). 